\title{
CARACTERÍSTICAS MORFOLÓGICAS DO LOBO DA ÍNSULA EM PACIENTES PORTADORES DE EPILEPSIA DO LOBO TEMPORAL MEDIAL
}

\author{
Feres Chaddad Neto ${ }^{1,2,3}$, Evandro de Oliveira ${ }^{1,2,3}$, Eric Paschoal ${ }^{1,3}$, \\ Fernando Cendes², Mário Santana Filho ${ }^{1,3}$
}

\begin{abstract}
RESUMO - A esclerose medial temporal (EMT) é caracterizada pela esclerose hipocampal e diferentes graus de acometimento das estruturas vizinhas como amígdala, giro parahipocampal e córtex entorrinal. 0 estudo avaliou 40 indivíduos com EMT e 40 do grupo controle. Os casos foram avaliados por um método para as medidas da ínsula (E-Film) e outro método para o cálculo do volume (Neuroline). Não houve diferença estatística de alteração de volume e das medidas do lobo da ínsula nos pacientes portadores de EMT. O estudo não demonstrou alteração morfológica da ínsula quando comparado os dois grupos.
\end{abstract}

PALAVRAS-CHAVE: esclerose medial temporal, ínsula, ressonância magnética.

\begin{abstract}
Morphological characteristics from the insula's lobe in patients with medial temporal lobe epilepsy
ABSTRACT - The temporal medial sclerosis (TMS) is characterized by hippocampal sclerosis in temporal and by distinghished grades of injury near to other neurological structures such as: amygdaloid nucleus, parahippocampal girus and entorhinal region. The study analyzed 40 patients with TMS and 40 people f rom the control cluster. All the cases were appreciated by one method for measurement of insula's cortex (E-Film) and another method to calculate the insula's volume (Neuroline). There is no variation statistical between the insula's volume and insula's measurement for the two clusters. This paper didn't show the insula's morphological variation when these two groups were compared.
\end{abstract}

KEY WORDS: temporal medial sclerosis, insula, magnetic resonance imaging.

A epilepsia do lobo temporal (ELT) é síndrome em que a atividade paroxística ictal inicia-se no lobo temporal (LT) e cujas crises epilépticas são caracterizadas por sensação epigástrica, alucinações olfatórias ou gustativas, "déja vu", "jamais vu", automatismos oroalimenta res, postura distônica de mão contralateral ao foco epileptogênico, alucinações visuais e confusão pós ictal proeminente ${ }^{1}$. A epilepsia do lobo temporal medial (ELTM) é o subtipo mais freqüente de ELT e a causa mais frequente é uma lesão estereotipada, de etiologia desconhecida, denominada esclerose medial temporal (EMT) (Fig 1). A EMT é caracterizada pela esclerose hipocampal e diferentes graus de acometimento das estruturas vizinhas como amígdala, giropara-hipocampal e córtex entorrinal. A importância clínica da ELTM dá-se pela sua alta prevalência e elevada proporção de pacientes que não respondem às diferentes drogas anti-epilépticas (DAEs), e que a p resentam um bom prognóstico com o tratamento cirúrgico ( 80 a $90 \%$ de probabilidade de controle de crises) $)^{2,3}$. Inicialmente, o arsenal diagnóstico para análise dos pacientes com ELTM resumia-se ao quadro clínico e aos estudos eletrofisiológicos (eletrencefalografia). Com o avanço tecnológico surgiram outros métodos de análise, dentre estes a ressonância magnética (RM) e o SPECT (ictal e interictal). A RM permite uma visualização macroscópica precisa e detalhada das estruturas mediais, tais como o hipocampo, bem como do restante do sistema nervoso central (SNC). Os sinais sugestivos de esclerose hipocampal identificados pela RM correlacionam-se de maneira positiva e precisa com achados eletrencefalográficos, clínicos, ne u ropsicológicos e neuropatológicos. Além disso o sucesso da terapia cirúrgica também parece depender dos achados de RM pré-operatórios ${ }^{47}$. Vários estudos mostraram que medidas volumétricas de estruturas

\footnotetext{
${ }^{1}$ Instituto de Ciências Neurológicas, São Paulo, Brasil; ${ }^{2}$ Departamento de Neurologia e Neurocirurgia da Universidade Estadual de Campinas (UNICAMP), Campinas, Brasil; ${ }^{3}$ Real e Benemérita Sociedade Portuguesa de Beneficência Hospital São Joaquim, São Paulo, Brasil.
}

Recebido 2 Dezembro 2005, recebido na forma final 30 Março 2006. Aceito 8 Maio 2006.

Dr. Feres Chaddad Neto - Praça Amadeu Amaral 27 / $5^{\circ}$ andar - 01327-010 São Paulo SP - Brasil. 


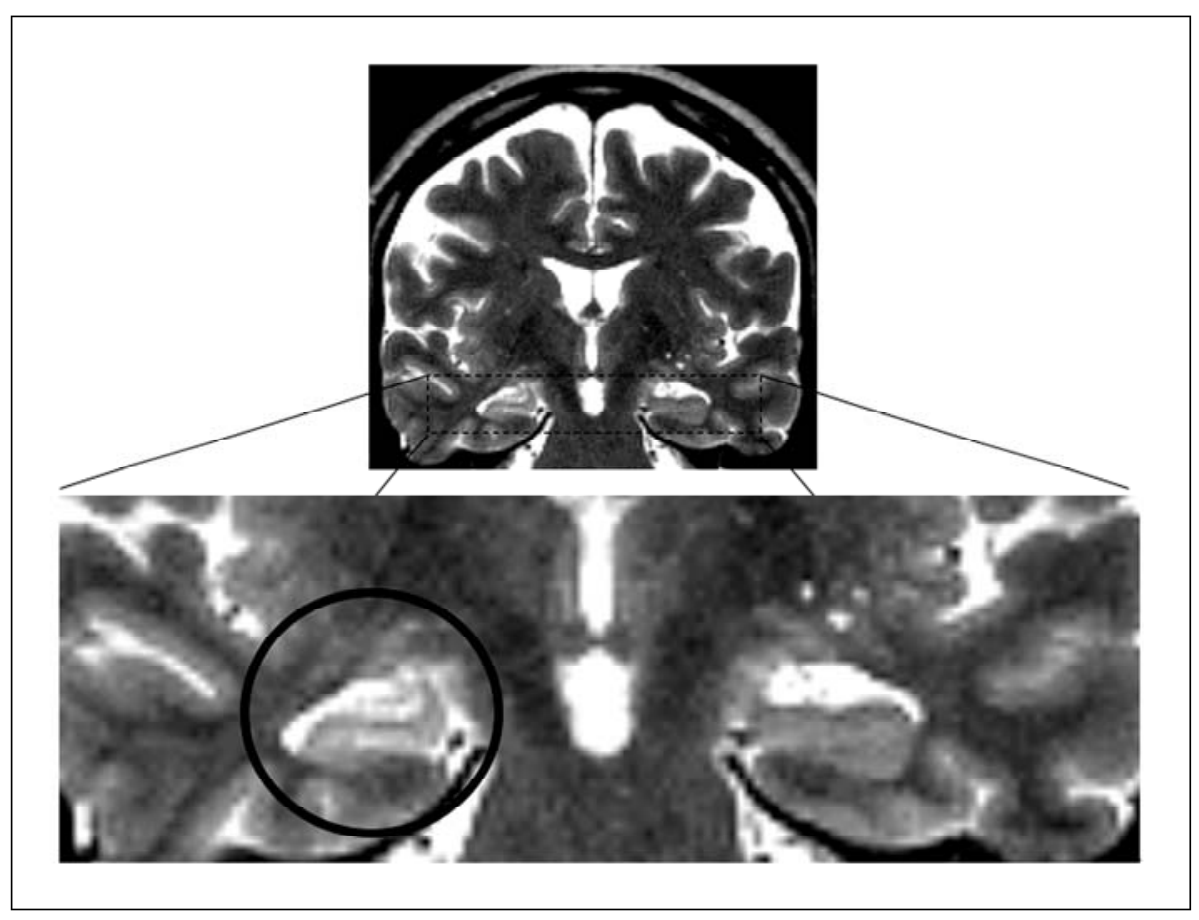

Fig 1. Esclerose hipocampal.

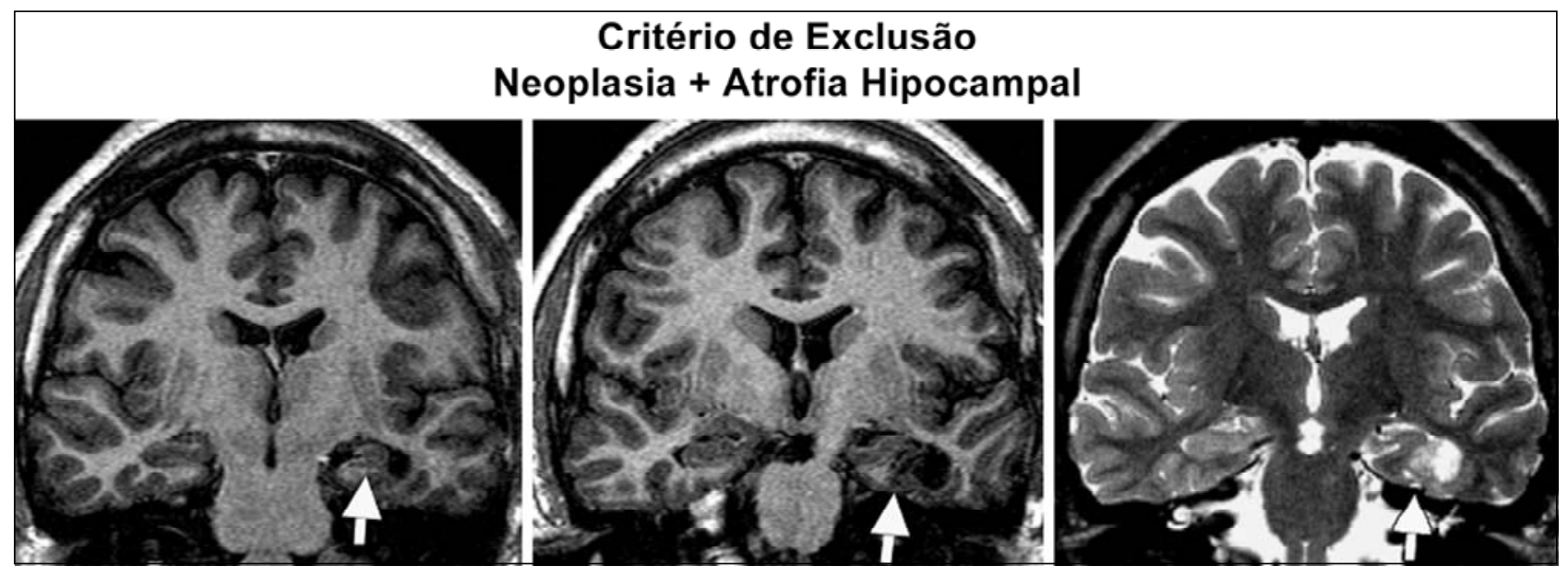

Fig 2. Atrofia hipocampal com patologia associada, corte coronal T1 e T2'.

do lobo temporal são capazes de detectar redução de volume de estruturas mediais que são pro po rcionais ao grau das alterações vistas no histopatológico e correlacionam com os resultados cirúrgicos ${ }^{4,8,9}$. Estudos volumétricos têm demonstrado lateralização précirúrgica correta em $80-90 \%$ dos pacientes com ELT, sem "falso positivos" 8 .

Do ponto de vista filogenético, a ínsula (Fig 2) é uma porção antiga do telencéfalo que persistiu durante a evolução e está presente em diversos espécimes, o que a torna alvo de estudos anatômicos comparativos. A identificação das características macro scópicas da ínsula foi, em sua maior parte, completada no final do século XIX². O lobo da ínsula (também conhecido com ilha de Reil) está localizado na profundidade dos lobos frontal, parietal e temporal. Apresenta o formato de pirâmide de base invertida, tendo como limite superior o sulco circular superior da ínsula, como limite anterior o sulco circular anterior da ínsula e como limite inferior o sulco circular inferior da ínsula ${ }^{3}$. Estudos subseqüentes foram direcionados à análise de suas conexões neuronais, suas características angiográficas, bem como seu papel na epilepsia. Mais recentemente, pesquisas sobre o papel funcional da ínsula tem sido direcionados para analisar suas relações com a linguagem, funções neurovegetativas e o córtex motor suplementar. Além disso, muita atenção tem sido voltada para o papel da ín- 
sula nas funções cardíacas, arritmias, picos hormonais, bem como na morte súbita entre pacientes com acidentes cerebrais isquêmicos e epilepsia ${ }^{9,11}$. A relevância desse estudo está baseada na possibilidade de se comparar a relação anatômica entre o lobo da ínsula e o lobo temporal de pacientes portadores de ELT, mais especialmente a ELMT. O lobo da ínsula, em dec o rrência de sua topografia apresenta amplas conexões com praticamente todas as partes do céreb ro através dos fascículos, que hoje estão sendo estudados na anatomia através da dissecação das fibras brancas em cérebros congelados e na RM do encéfalo através da tratografia ${ }^{4,12}$. Essas conexões começam a justificar as relações das linguagens, funções neurovegetativas e o córtex motor suplementar ${ }^{5}$.

O presente estudo é único, e evidencia os aspectos morfológicos, topográficos e, principalmente, volumétricos do lobo da ínsula através da RM tanto dos indivíduos normais quanto dos pacientes port a do res de ELMT, tem como objetivo compará-los na busca minuciosa de alterações anatômicas ipsilaterais à esclerose medial temporal nos pacientes com ELMT, permitindo correlacionar muitas das manifestações clínicas dos pacientes port a do res desta entidade nosológica com o comprometimento do lobo da ínsula e suas vias. Em decorrência da proximidade e conexão do lobo da ínsula com o lobo temporal, das manifestações clínicas sugestivas de envolvimento de lobo da ínsula na ELTM, realizamos um estudo das alterações anatômicas da ínsula em pacientes com EMT. O trabalho visa analisar a anatomia do lobo da ínsula (morf ol ogia, volume e topografia) de indivíduos portadores de ELTM; verificar se há alteração do volume e medida do lobo da ínsula em indivíduos portadores de ELTM, utilizando os programas Neuroline e E-Film, respectivamente; e verificar se há relação entre volume do lobo da ínsula e sinais de esclerose hipocampal.

\section{MÉTODO}

O estudo foi realizado de forma prospectiva e descritiva, através da análise de RM de indivíduos adultos selecionados no Ambulatório de Epilepsia do Hospital das Clínicas da UNICAMP e no setor de neuroimagem da MED IMAGEM do Hospital Real e Benemérita Sociedade Portuguesa de Beneficência de São Paulo (HRBSPBSP).

Do Ambulatório de Epilepsia do Hospital das Clínicas da UNICAMP, selecionamos 20 pacientes portadores de ELTM com esclerose hipocampal identificada por RM; o diagnóstico foi estabelecido através da avaliação clínica por neurologistas experientes, estudo eletrencefalográfico e alteração do hipocampo à RM de encéfalo em um sistema de 2 Teslas. As alterações do hi pocampo foram baseadas em alterações de sinal, volume e formato nesse sistema de RM.
O grupo controle foi formado por 20 indivíduos normais, sem história prévia de doenças envolvendo o SNC.

No setor da neuroimagem da MED IMGEM do HRBSPBSP, selecionamos 20 indivíduos portadores de ELTM com esclerose medial temporal, o diagnóstico foi estabelecido através da avaliação clínica por neurologistas, estudo eletrencefal ográfico e alteração do hipocampo à RM, utilizando o aparelho de 1,5 teslas (CVI, LX, EXCITE 1 e EXCITE 2). Nos pacientes selecionados na UNICAMP, a análise volumétrica do lobo da ínsula por RM foi realizada utilizando o programa de segmentação de imagem designado Neuroline, no laboratório de neuroimagem. Este programa permitiu o estabelecimento da comparação entre os indivíduos normais e os indivíduos com esclerose medial hipocampal em relação a volumetria do lobo da ínsula.

Estabeleceu-se a comparação entre o volume do lobo da ínsula ipsilateral à esclerose medial hipocampal e o volume do lobo da ínsula contralateral.

Foi analisado o volume do lobo da ínsula de ambos os hemisférios cerebrais no plano sagital, na seqüência em T1, no método neuroline versão 0,2 , pelo método de segmentação semi-automática baseada no método Watershal. Utilizou-se a segmentação das imagens DICOM em plano 2D, utilizando um sistema de cores de linhas em que a cor vermelha delimita a área a ser contado o volume e a cor a ma rela estima o volume do lobo da ínsula. A análise detalhada do volume do lobo da ínsula nas imagens de RM de 2 Teslas consiste da seguinte forma: transposição das imagens de RM para o programa de segmentação de imagem Neuroline; identificação dos limites do lobo da ínsula; separação do lobo da ínsula das demais porções do encéfalo por um sistema de cores; cálculo do volume. Realizou-se a transposição das imagens de RM de 40 pacientes do Hospital das Clínicas da UNICAMP para o programa de segmentação de imagem Neuroline, sendo 20 indivíduos sem patologia prévia e 20 com o diagnóstico de ELTM.

Selecionou-se as imagens sagitais em $\mathrm{T} 1$ ponderadas "spin echo", onde se verifica toda a extensão do lobo da ínsula.

A segunda etapa consistiu na identificação dos limites do lobo da ínsula: limite anterior: sulco circular anterior, limite superior: sulco circular superior e limite inferior: sulco circular inferior.

A terceira etapa consistiu em delinear o lobo da ínsula através de um sistema de cores, onde se padronizou colorir os limites com coloração amarela e o parênquima com coloração vermelha, sendo a opção aleatória.

A quarta etapa consistiu na leitura do volume da estrutura colorida em vermelho, ou seja, a ínsula, fornecida diretamente pelo Neuroline (Fig 3).

Nos pacientes selecionados no setor da MED IMAGEM/ HRBSBPSP, o E-film foi o programa de análise de imagens que permitiu o cálculo da extensão das linhas dos sulcos circulares anterior, superior e inferior. Com isso pôde ser feita as medidas de forma indireta do lobo da ínsula, com seqüências em T1 no plano sagital. Analisou-se o giro insular longo anterior do lobo da ínsula na imagem sagital em T1. Estabeleceu-se a comparação das medidas do lobo da ínsula entreindivíduos normais e portadores da esclerose medial hipocampal. Comparou-se as medidas do lobo da ínsula 


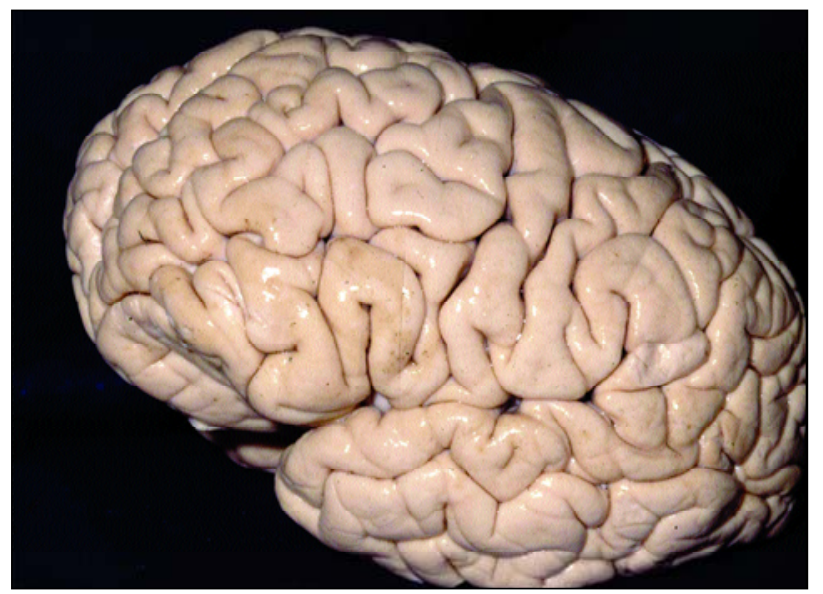

Fig 3. Lobo temporal.

ipsilateral à esclerose medial hipocampal com as medidas do lobo da ínsula contralateral.

A análise detalhada do lobo da ínsula nas imagens de RM de 1,5 Teslas consistiu da seguinte forma: transposição das imagens de RM para o programa E- Film; identificação dos limites do lobo da ínsula, calculando a extensão desses limites; identificação do giro insular longo anterior com sua posterior medida, sendo esta perpendicular à angulação do giro no plano sagital T1. Realizou-se a transposição de imagens de 40 pacientes da MED IMAGEM do Hospital Beneficência Portuguesa de São Paulo para o programa EFilm, sendo 20 indivíduos sem patologia prévia e 20 com o diagnóstico de ELTM decorrente de EMT. Selecionou-se as imagens sagitais em T1 ponderadas "spin echo", onde se verifica toda a extensão do lobo da ínsula. A segunda etapa consiste na identificação dos limites do lobo da ínsula: limite anterior: sulco circular anterior, limite superior: sulco circular superior e limite inferior: sulco circular inferior (Fig 4).

A terceira etapa consiste na marcação dos sulcos circula res com leitura dos seus resultados. Anterior: a linha traçada do ângulo ântero-superior ao ângulo ântero-inferior (límen da ínsula). Superior: linha traçada do ângulo ânterosuperior ao ângulo posteror. Inferior: linha traçada do ângulo posterior ao límen da ínsula (Fig 5).

\section{RESULTADOS}

Para o Neuroline-Entre os pacientes com ELTM com EMT, 9 eram mulheres (45\%) e 11 homens (55\%). A idade média foi 37,4 anos $( \pm 9,32$ anos), variando de 17 a 55 anos. O volume do lobo da ínsula variou de 18321 a $23401 \mathrm{~mm}^{3}$ à esquerda e de 18010 a 23604 $\mathrm{mm}^{3}$ à direita.

O volume do lobo da ínsula direita ipsilateral à EMT variou de 18102 a $19905 \mathrm{~mm}^{3}$ e o volume da ínsula contralateral variou de 18331 a $20109 \mathrm{~mm}^{3}$.

$\mathrm{O}$ volume da ínsula esquerda ipsilateral à EMT variou de 18321 a $23401 \mathrm{~mm}^{3}$ e o volume da ínsula contralateral variou de 18010 a $23604 \mathrm{~mm}^{3}$.

Entre os pacientes do grupo controle, 12 eram mulheres $(60 \%)$ e 8 eram homens (40\%). A idade mé-

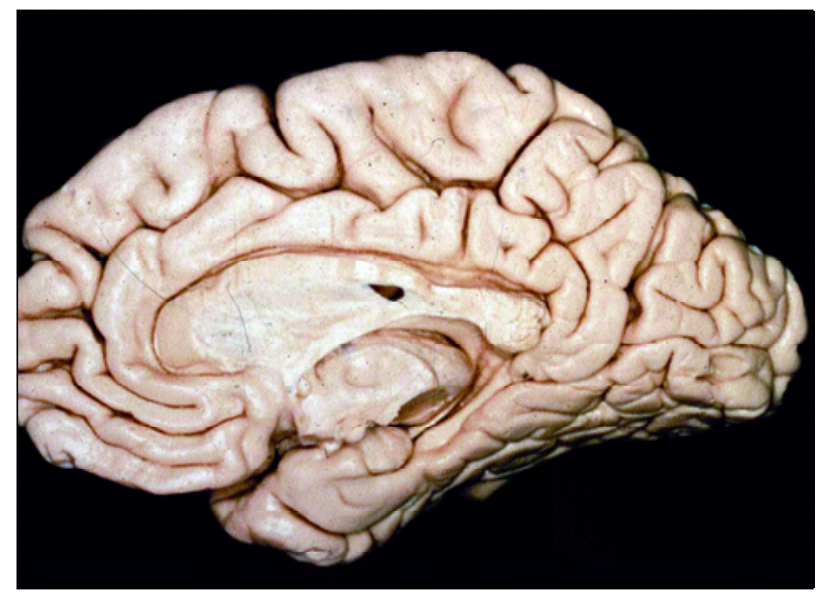

Fig 4. Lobo límbico.

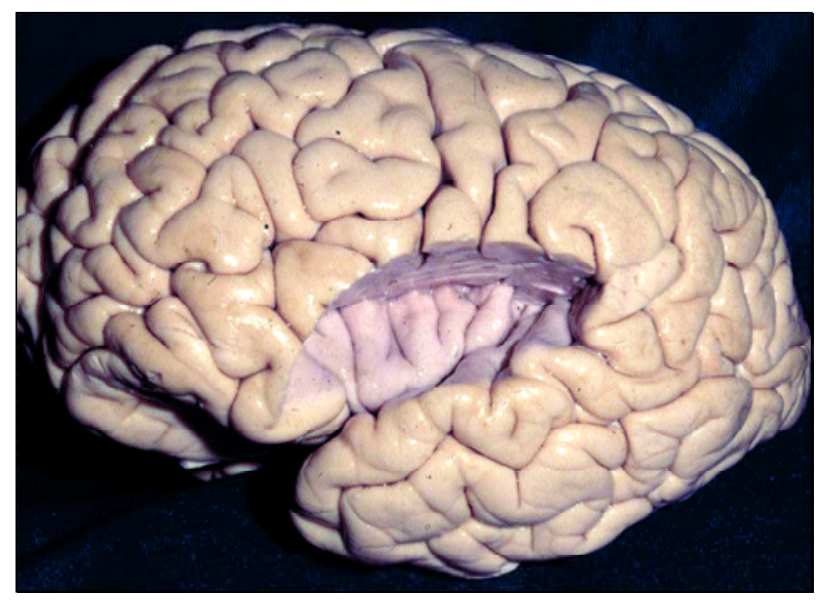

Fig 5. Lobo da ínsula.

Tabela 1. Comparação entre os grupos esclerose e controle Neuroline.

Testes estatísticos ${ }^{\mathrm{b}}$

\begin{tabular}{lcc} 
& IE_Neuro & ID_Neuro \\
\hline Mann-Whitney U & 194.500 & 179.000 \\
Wilcoxon W & 404.500 & 389.000 \\
Z & -.149 & -.568 \\
Asymp. Sig. (2-tailed) & .882 & .570 \\
Exact Sig [2*(1-tailed Sig.)] & $.883^{\mathrm{a}}$ & $.583^{\mathrm{a}}$ \\
\hline anão corrigido; ${ }^{\mathrm{b}}$ grupos. & &
\end{tabular}

Tabela 2. Comparações entre os lados ( $D$ e E ou Lesão e Oposto) para o grupo esclerose.

\begin{tabular}{lcc}
\hline \multicolumn{3}{c}{ Testes estatísticos $^{\mathrm{b}}$} \\
& $\begin{array}{ll}\text { ID_Neuro } \\
\text { IE_Neuro }\end{array}$ & $\begin{array}{c}\text { IO_Neuro } \\
\text { IL_Neuro }\end{array}$ \\
\hline $\mathrm{Z}$ & $-.989^{\mathrm{a}}$ & $-.429^{\mathrm{a}}$ \\
Asymp. Sig. (2-tailed) & .322 & .668 \\
\hline apositivo; b'Wilcoxon Signed Ranks Test.
\end{tabular}


Tabela 3. Medidas descritivas das variáveis avaliadas pelo método E-Film.

\begin{tabular}{|c|c|c|c|c|c|c|c|}
\hline Variável & Grupo & $\mathrm{n}$ & Mínimo & Máximo & Mediana & Média & DP \\
\hline \multirow[t]{2}{*}{ SCAE } & Controle & 20 & 2,00 & 3,00 & 2,40 & 2,47 & 0,30 \\
\hline & Esclerose & 20 & 2,30 & 2,80 & 2,50 & 2,52 & 0,17 \\
\hline \multirow[t]{2}{*}{ SCAD } & Controle & 20 & 1,90 & 2,90 & 2,45 & 2,46 & 0,31 \\
\hline & Esclerose & 20 & 2,20 & 2,80 & 2,40 & 2,47 & 0,17 \\
\hline SCA_L & Esclerose & 20 & 2,20 & 2,70 & 2,40 & 2,46 & 0,17 \\
\hline SCA_O & Esclerose & 20 & 2,30 & 2,80 & 2,50 & 2,54 & 0,16 \\
\hline \multirow[t]{2}{*}{ SCSE } & Controle & 20 & 2,50 & 5,60 & 4,10 & 4,27 & 0,91 \\
\hline & Esclerose & 20 & 2,50 & 5,40 & 4,65 & 4,57 & 0,79 \\
\hline \multirow[t]{2}{*}{ SCSD } & Controle & 20 & 2,20 & 5,60 & 4,20 & 4,26 & 0,92 \\
\hline & Esclerose & 20 & 2,40 & 5,50 & 4,65 & 4,55 & 0,81 \\
\hline SCS_L & Esclerose & 20 & 2,40 & 5,30 & 4,65 & 4,52 & 0,80 \\
\hline SCS_O & Esclerose & 20 & 2,50 & 5,50 & 4,65 & 4,60 & 0,79 \\
\hline \multirow[t]{2}{*}{$\mathrm{SCIE}$} & Controle & 20 & 3,20 & 4,60 & 3,60 & 3,80 & 0,54 \\
\hline & Esclerose & 20 & 3,20 & 4,50 & 4,10 & 3,88 & 0,51 \\
\hline \multirow[t]{2}{*}{ SCID } & Controle & 20 & 3,10 & 4,40 & 3,75 & 3,79 & 0,43 \\
\hline & Esclerose & 20 & 3,10 & 4,50 & 4,15 & 3,85 & 0,51 \\
\hline SCl_L & Esclerose & 20 & 3,10 & 4,40 & 4,15 & 3,83 & 0,52 \\
\hline SCl_O & Esclerose & 20 & 3,20 & 4,50 & 4,10 & 3,90 & 0,49 \\
\hline \multirow[t]{2}{*}{ GIAPE } & Controle & 20 & 1,40 & 2,00 & 1,60 & 1,66 & 0,17 \\
\hline & Esclerose & 20 & 1,50 & 1,90 & 1,65 & 1,68 & 0,14 \\
\hline \multirow[t]{2}{*}{ GIAPD } & Controle & 20 & 1,50 & 1,80 & 1,65 & 1,65 & 0,07 \\
\hline & Esclerose & 20 & 1,50 & 1,90 & 1,70 & 1,70 & 0,12 \\
\hline GIAP_L & Esclerose & 20 & 1,50 & 1,90 & 1,70 & 1,68 & 0,12 \\
\hline GIAP_O & Esclerose & 20 & 1,50 & 1,90 & 1,65 & 1,70 & 0,14 \\
\hline \multirow[t]{2}{*}{ GISIE } & Controle & 20 & 3,50 & 4,00 & 3,60 & 3,69 & 0,16 \\
\hline & Esclerose & 20 & 3,50 & 4,00 & 3,70 & 3,73 & 0,16 \\
\hline \multirow[t]{2}{*}{ GISID } & Controle & 20 & 3,60 & 3,90 & 3,70 & 3,68 & 0,07 \\
\hline & Esclerose & 20 & 3,60 & 4,00 & 3,70 & 3,73 & 0,12 \\
\hline GISI_L & Esclerose & 20 & 3,60 & 3,90 & 3,70 & 3,71 & 0,11 \\
\hline GISI_O & Esclerose & 20 & 3,50 & 4,00 & 3,70 & 3,75 & 0,17 \\
\hline
\end{tabular}

SCAE (sulco circular anterior esquerdo); SCAD (sulco circular anterior direito); SCA_L (sulco circular anterior da lesão); SCA_O (sulco circular anterior oposto); SCSE (sulco circular superior esquerdo); SCSD (sulco circular superior direito); SCS_L (sulco circular superior da lesão); SCS_O (sulco circular superior oposto); SCIE (sulco circular inferior esquerdo); SCID (sulco circular inferior direito); SCI_L (sulco circular inferior da lesão); SCI_O (sulco circular inferior oposto); GIAPE (giro insular longo antero-posterior esquerdo); GIAPD (giro insular longo antero-posterior direito); GIAP_L (giro insular longo antero-posterior da lesão); GIAP_O (g i ro insular longo antero-posterior oposto); GISIE (giro insular longo súpero-inferior esquerdo); GISID (giro insular longo súperoinferior direito); GISI_L (giro insular longo súpero-inferior da lesão); GISI_O (giro insular longo súpero-inferior oposto).

dia foi 35,7 anos $( \pm 8,96)$, de 24 a 54 anos. O volume do lobo da ínsula esquerda variou de 17044 a 22418 $\mathrm{mm}^{3}$, e para o lobo da ínsula direita os valores variaram de 17210 a $22313 \mathrm{~mm}^{3}$.

As comparações dos grupos controle e esclerose foram realizadas através do teste de Mann-Whitney. Para comparação das variáveis avaliadas do lado oposto e de mesmo lado da lesão ou dos lados direito e esquerdo para o grupo da esclerose, foi adotado o teste de Wilcoxon para amostras pareadas.

A Tabela 1 mostra os resultados das comparações entre os grupos esclerose e controle (o valor de $p$ encontra-se na linha "Exact Sig..."). A Tabela 2 apresenta os resultados das comparações entre os lados dire ito e esquerdo ou lado da lesão e o oposto para o grupo e scle rose(o valor de $p$ encontra-se na linha "Asymp. Sig...").

Para o E-Film - O grupo controle é composto de 10 mulheres $(50 \%)$ e de 10 homens $(50 \%)$ com idade média de 33,8 anos $( \pm 12,11)$, variando de 18 a 59 anos. O grupo esclerose é composto de 10 mulhere $\mathrm{s}$ (50\%) e 10 homens (50\%) com idade média de 37,1 anos $( \pm 15,75)$, variando de 12 a 59 anos.

A Tabela 3 descreve as medidas das variáveis avaliadas pelo método E-Film. O resultado das comparações entre os dois grupos mostrou valores de $\mathrm{p}$ sem significância estatística pelos métodos. 


\section{DISCUSSÃO}

Os avanços da neuroimagem permitiram a identificação in vivo de alterações da estrutura cere bral antes identificada apenas por exames anatomopatológicos. Com o advento da RM, a correlação entre achados de imagem e análise de espécimes cirúrgicos tornou-se realidade, principalmente no caso da ELTM. Alguns achados de RM - como a atrofia hipocampal - passaram a ser utilizados como achado patológico correlato, ou seja, a esclerose hipocampal?.

Posteriomente, os avanços no pós-processamento da imagem permitiu que novas técnicas fossem desenvolvidas. A volumetria de hipocampo permite a identificação de alterações sutis que não podem ser identificadas pela análise visual de rotina, mesmo tratando-se de neuroradiologista experiente ${ }^{13-15}$.

Mais recentemente, estudos de segmentação da imagem que demonstraram que pacientes com ELTM também apresentam o envolvimento de estruturas como o córtex fronto-orbital, em especial o giro reto e o giro orbital medial, ambos associados à projeção monossináptica da região hipocampal CA $1^{12}$. Outras áreas também se mostram acometidas como o tálamo, compreendendo os núcleo mediodorsal e o pulvinar $^{16-18}$. Uma possível explicação para esse achados é o fato dessas áreas apresentarem conexão com est ruturas do lobo temporal medial, incluindo o hipocampo ${ }^{13}$. Assim como o núcleo médiodorsal do tálamo apresenta conexão com o córtex entorrinal ${ }^{13}$.

O lobo da ínsula se apresenta topograficamente próximo e com múltiplas conexões com o lobo temporal medial ${ }^{14}$. Além disso, as crises epilépticas geradas na ínsula apresentam semiologia semelhante às crise originadas nas estruturas mediais temporais.

Apesar da inter-relação da ínsula com o lobo temporal medial, nosso estudo não demonstrou a presença de alteração da volumetria da ínsula nos indivíduos port a d o res de EMT quando comparados com o lado contralateral à esclerose, bem como quando comparado com o grupo controle. A análise estatística se baseou em dois métodos para avaliação das medidas de volume da ínsula (NEUROLINE) e de contorno da área que as margens do lobo da ínsula ocupa em sua topografia (E-FILM).

Apesar de tratar-se de estudo preliminar e da necessidade da avaliação de um número maior de pacientes para que os achados sejam confirmados, aparentemente o volume da ínsula em pacientes com ELTM é pres e rado a despeito do que se observa nas outras estruturas do sistema límbico.
Em conclusão, o estudo das características morf ológicas do lobo da ínsula em pacientes portadores de ELTM decorrente de EMT, mostrou que: o lobo da ínsula não apresentou alteração do volume à ressonância magnética de alta resolução, utilizando o programa Neuroline, nos port a d o res de EMT; o lobo da ínsula não apresentou alteração de formato, espessura de seus giros e comprimento de seus limites à RM convencional utilizando o programa E- Film nos pacientes portadores de EMT; no momento, nenhum dos métodos conseguiu demonstrar alteração anatômica do lobo da ínsula à RM, mesmo que sutis, nos pacientes portadores de ELTM decorrente de EMT.

\section{REFERÊNCIAS}

1. Olivier A, Tanaka T, Andermann F. Reoperations in temporal lobe epilepsy. Epilepsia 1998;29:678

2. Gray's Anatomy. $36^{\text {th }}$ British Edition: Williams \& Warwick (eds). New York: Saunders, 1980:987-988.

3. Rhoton AL Jr. The cerebrum. In Rhoton's anatomy. Neurosu rgery 2003; 53:41.

4. Duffau H, Capelle L, Lopes M, Faillot T, Sichez JP, Fohanno D. The insular lobe: physiopathological and surgical considerations. Neurosurgery 2000;47:801-811.

5. Cendes F, Andermann F, Dubeau F, et al. Early childhood prolonged febrile convulsions, atrophy and sclerosis of mesial structu res and temporal lobe epilepsy: an MRI volumetric study. Neurology 1993;43: 1083-1087.

6. Briton CJ. The Neuropathology of temporal lobe epilepsy. Oxford: Oxford University Press, 1988;1-158.

7. Commision on Classification and Terminology of the International League Against Epilepsy, Proposal for revised clinical and electroencephalographic classification of epileptic seizures. Epilepsia 1981;22: 489-501.

8. Engel J Jr. Recent advances in surgical treatment of temporal lobe epilepsy. Acta Neurol Scand 1992;86(Suppl 140):S71-S80.

9. Engel J Jr, Williamson PD, Wieser H-G. Mesial temporal lobe epilepsy. In Engel J Jr, Pedley TA (eds). Epilepsy: a comprehensive textbook. Philadelphia: Lippincott-Raven, 1997:2417-2426.

10. Jack CR, Sharbrough FW, Cascino GD, et al. Magnetic resonance imagebased hippocampal volumetry: correlation with outcome after temporal lobectomy. Ann Neurol 1992;31:138-146.

11. Nauta WJH, Feirtag M. A ffect and motivation: the limbic system. In Fundamental neuroanatomy. New York: WH Freeman \& Co, 1986: 120-127.

12. Nauta WJH, Feirtag $M$, Axon tracing. In Fundamental neuroanatomy. New York: WH Freeman \& Co, 1986:50-61.

13. Amaral DG, Witter MP. The three-dimensional organization of the hippocampal formation: a review of anatomical data. Neuroscience 1989;31:571-591.

14. Arruda F, Cendes F, Andermann F, et al. Mesial atrophy and outcome after amygdalohippocampectomy or temporal lobe removal. Ann Neurol 1996;40:446-450.

15. Barbas H, Blatt GJ. Topographically specific hippocampal projections target functionally distinct prefrontal areas in the rhesus monkey. Hippocampus 1995;11:511-533.

16. Bentivolgio M, Aggleton JP, Mishkin M. The thalamus and memory formation. In Steriade EGJ, McCormick DA (eds). Thalamus: experimental and clinical aspects. Amsterdam: Lausanne, 1997:869-871.

17. Tü reU, Yasargil DCH, Al-Mefty O, Yasargil MG. Topografhic anatomy of the insular region. J Neurosurg 1999;90:720-733.

18. Cendes F, Caramanos Z, Andermann F, et al. Próton magnetic resonance spet roscopic imaging and magnetic resonance imaging volumetry in the lateralization of temporal lobe epilepsy: a series of 100 patients. Ann Neurol 1997;42:737-746. 